\title{
A Low-Cost Multichannel Optical Transmission System for Video Signals
}

\author{
L. A. Dick van den Broeke, Leo P. de Jong, Jan Davidse, and Arthur H. M. van Roermund
}

\begin{abstract}
A novel low-cost multichannel optical transmission system for video signals is described. The system uses time-division multiplex pulse-position modulation (TDPPM). The transmitter and receiver are realized in a standard bipolar process while an external low-cost laser and PIN photodiode are used. A maximum of 16 channels can be multiplexed, while achieving a weighted signal-to-noise ratio (SNR) of $54 \mathrm{~dB}$, a differential gain of $1 \%$ and a differential phase of $0.5^{\circ}$. For a launched power of $0 \mathrm{dBm}$ (pulse amplitude), the optical budget for a $45 \mathrm{~dB}$ weighted SNR exceeds $20 \mathrm{~dB}$. Besides video signals the system is also capable of handling other signals such as digital coded audio signals or computer data. The feasibility of a four-channel system has been confirmed by measurements.
\end{abstract}

\section{INTRODUCTION}

$\mathbf{O}$ PTICAL video transmission systems are being applied in a steadily growing field of local applications in which distances up to a few kilometers have to be covered. In several of these applications, for example in the surveillance of buildings, subway stations or bridges, high performance is not of primary concern. A weighted signal-to-noise ratio (SNR) of $50 \mathrm{~dB}$ (for PAL coded video), a differential gain of $2 \%$ and a differential phase of $1^{\circ}$ are generally satisfactory. For these applications the costs of the electro-optic transmitters and opto-electric receivers must be kept low because of the relatively low costs of other equipment, such as the fiber, the cameras and the monitors.

To cut the costs, there are two basic strategies: 1) Keep the actual costs of the transmitter and receiver as low as possible, and 2) use multiplexing techniques to reduce the relative costs of the fiber, transmitter, receiver and installation.

Up to now, most of the optical transmission systems presented by various authors have concentrated on (highperformance) single-channel systems [1]-[3] or high-cost multichannel systems [4]-[6]. In this paper, we present a novel low-cost multichannel system based on analog pulse-position modulation (PPM) and time-division multiplexing (TDPPM). With this coding scheme, the system becomes insensitive to non-linearities of the optical transmitter, which makes it possible to use low-cost LED's or laser diodes. Another advantage of the TDPPM system is that the transmitter and receiver circuitry can be integrated in a standard low-cost bipolar IC process with a minimum number of external

Paper approved by L. G. Kazovsky, the Editor for Optical Communications of the IEEE Communications Society. Manuscript received May 5, 1992; revised April 21, 1994

The authors are with the Delft University of Technology, Department of Electrical Engineering, Mekelweg 4, 2628 CD Delft, The Netherlands.

IEEE Log Number 9413166 components and adjustments. Compared to systems based on digital coding, the TDPPM system requires considerably less chip area and supply power.

In Section II we will explain why the TDPPM coding scheme is the most suitable for a low-cost system. Section III will deal with the architectures of the TDPPM transmitter and the receiver. In Section IV we will examine some important characteristics of low-cost optical components and the receiver front-end and determine the relations between the SNR, number of multiplexed channels and the available technology. In addition, we will investigate the influence of optical reflections and compare TDPPM coding with digital coding schemes. We will conclude by presenting some experimental results in Section V.

\section{TIME-Division Multiplex PPM CODING}

Various alternative digital and analog coding schemes are currently being applied in communication systems. In pursuing a minimum-cost system, we concluded, based on the following considerations, that analog TDPPM is preferable.

Since the approaches to designing systems based on digital coding are completely different from those based on analog alternatives, a first decision to be made is between analog and digital coding.

Digital systems have two important advantages over analog systems: 1) once the signals are in digital format, there are no impairments of the signal quality (which is not a strong argument because repeaters are not necessary) and 2) since standard configurations of the required cells are available, the design is much easier. On the other hand, digital systems have two important disadvantages when compared with analog systems: 1) since a relatively large number of gates is required to implement a specific function and since their switching times must be relatively short, the total power consumption and the chip area will be considerably larger, and 2) for wideband signals exhibiting a high dynamic range, the conversion from analog to digital (ADC) and from digital to analog (DAC) becomes increasingly difficult. Hence, an important advantage of an analog system is that its cost will be less than that of a digital system.

Another important criterion is the quality of the transmitted signals. As will be shown in Section IV-F digital systems achieve an SNR which is comparable to that achieved by the system based on analog TDPPM coding.

Finally, we should bear in mind that, if we choose the analog solution, this does not imply that only analog signals can be 


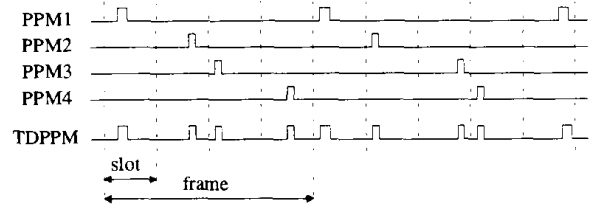

Fig. 1. The waveforms of 4 PPM signals multiplexed into a TDPPM signal. The vertical dashes denote the boundaries of the time slots.

handled. Due to its transparency, the system is capable of transmitting digital signals as well.

In conclusion, the analog solution is preferred because of its relatively low cost.

Since typical low-cost LED's and laser diodes suffer from amplitude distortions in the order of 5\% [7], laser non-linearity is a decisive factor in choosing an appropriate analog coding scheme. First, we opt for time-division multiplexing because in frequency-division multiplex systems, the resulting intermodulation and harmonic distortions cause severe crosstalk between channels. Second, the video signal has to be coded in the phase of a carrier signal or in the positions of pulses or pulse edges to avoid distortion of the video signal. Since in our system the modulation scheme should be compatible with time-division multiplexing, pulse modulation is a natural choice.

Since bandwidth-limitations of the transmission channel in addition to non-linearities lead to inaccuracies in the measured positions or width of the pulses, the signals should preferably be carried by the positions of pulses which are equally shaped and of equal amplitude. Hence, we end up with (time division multiplex) pulse position modulation.

In a PPM modulator, the input signal is sampled and converted into pulses of which the timing, relative to their zero-reference positions, is proportional to the sampled values. In TDPPM coding, each of the video input signals is converted by a separate PPM modulator. Since the resulting PPM signals have their own zero-reference positions and time slots (Fig. 1), multiplexing is performed by simply adding the individual pulse trains. One complete series of pulses, one pulse for each channel, is called a frame. The pulses of one of the channels (PPM1) are slightly widened. These so called "frame pulses", will be identified by the receiver so that the receiver knows when a new frame starts (this will be discussed in Section III-B).

In conclusion, TDPPM is the most suitable coding. It features multiplexing and is insensitive to the non-linearities of low-cost lasers. Moreover, the entire transmitter and receiver can be integrated on a minimum of chip area.

\section{SYSTEM ARCHITECTURE}

In this section, we present the global architectures of the TDPPM transmitter and receiver, which include the electronic circuits as well as the optical components.

\section{A. Transmitter}

A straightforward configuration of the transmitter is depicted in Fig. 2. We distinguish the following functions.

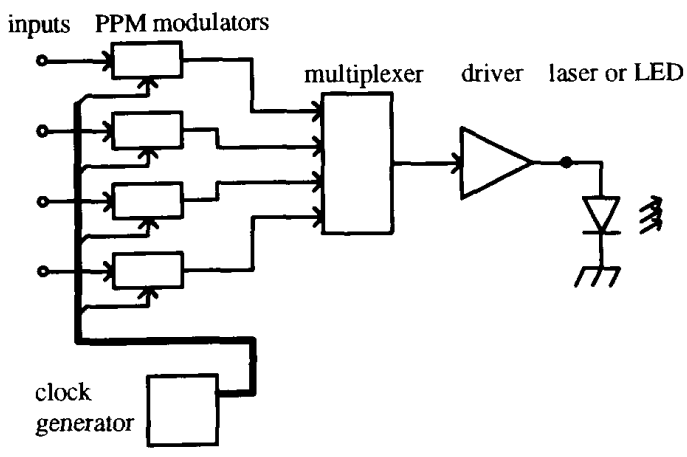

Fig. 2. Transmitter architecture.

- Modulator: Samples the input signal and converts the samples into PPM pulses. The modulators are synchronized by a clock signal in such a way that the PPM pulses are already in the appropriate time slots.

- Multiplexer: Adds the PPM pulses together into one TDPPM signal (see Fig. 1). As the PPM pulses are already in the appropriate slots, the multiplexing function may consist of a simple summing circuit.

- Clock: Provides the (multiphase) synchronization signal for the modulators.

- Output Driver: Amplifies TDPPM pulses to the level required for properly driving the LED or laser and provides its biasing.

- LED or Laser Diode: External (that is, not integrated in the IC) lightwave transmitter, mounted in a fiber-optic connector or pig-tailed with a piece of optical fiber.

Rather than locating the multiplexer at the outputs of the modulators, it would seem preferable to locate the multiplexer at the transmitter inputs instead, so only one modulator is required. However, our solution has two important advantages: 1) signal distortion due to non-linearity of the multiplexer switch is avoided (the low-cost IC process does not feature MOS switches) and 2) in between the generation of two successive TDPPM pulses, the modulators is left a relatively long "recovery" time. Sufficiently long recovery time is necessary to avoid modulator distortion. The alternative solution would not provide the feature. After, for example, a PPM pulse of signal 1 has been transmitted at the end of the time slot, the next PPM pulse, belonging to signal 2, may already appear at the start of the next slot.

\section{B. Receiver}

Fig. 3 depicts the block diagram of the receiver. It consists of the functions:

- Photodiode: PIN optical receiver, mounted in a fiber-optic connector or pig-tailed with a piece of optical fiber.

- Amplifier: Amplifies the TDPPM pulses received by the photodiode [9], [10]. Additionally, it performs the receiver low-pass filtering before the pulses are supplied to the pulse reshaper [see Section IV-C1)]. Although the amplitude of the received TDPPM pulses is not fixed due to different coupling and fiber losses, the gain of the 


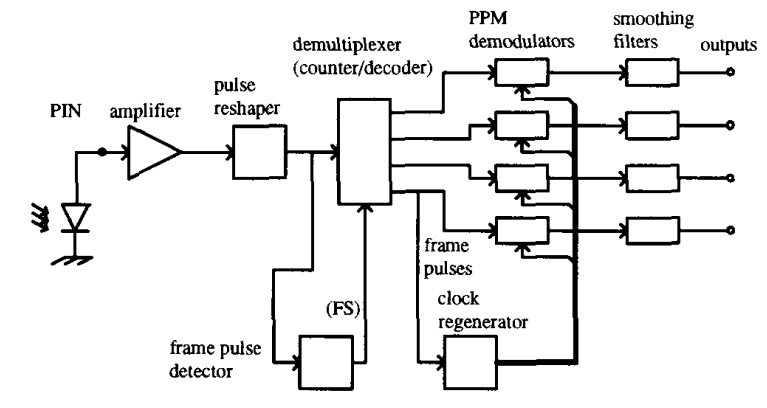

Fig. 3. Receiver architecture.

amplifier need not be variable because the pulse reshaper can handle a large range of input pulse amplitudes.

- Pulse Reshaper: Reconstructs proper "square" pulses with constant amplitude and shape and minimal pulse jitter. In this way, the quality (i.e., the SNR) of the received signals only depends on the performance of the pulse reshaper and not on that of the demultiplexer and the demodulator.

- Frame Pulse Detector: Detects frame pulses (which are a little wider) from other TDPPM pulses, necessary for proper synchronization of the demultiplexer.

- Demultiplexer: Splits the TDPPM pulse train into the individual PPM pulse trains, one for each channel. The demultiplexer requires frame synchronization (FS), supplied by the frame pulse detector, and slot synchronization, which is derived directly from the TDPPM pulses.

- Demodulator: Converts the PPM pulses into duration modulated (PDM) pulses so that the baseband signal can be retrieved by low-pass filtering. The time reference, indicating the zero positions of the PPM pulses, is supplied by the clock regenerator.

- Low-Pass Smoothing Filter: Removes high frequency signals consisting of sidebands around the sample frequency and its harmonics from the PDM signal. The baseband signal is available at the filter output. We used the integrated video filter described in [12].

- Clock Regenerator: Derives the multiphase clock signal required for demodulation from the frame pulses that are available at one of the demultiplexer outputs.

Since TDPPM pulses can be located at any position in their time slots, proper operation of the demultiplexer does not allow delay errors between the TDPPM pulses and the signal determining the slot synchronization of the demultiplexer. To avoid delay errors, the demultiplexer is synchronized directly by the TDPPM pulses themselves: The state of the demultiplexer is altered after a complete TDPPM pulse has been received.

A principal disadvantage of this technique is its sensitivity to false pulses (which appear if the SNR of the received pulses is below a certain level, see Section IV-D). Concerning this sensitivity, a better solution would be to have the slot synchronization signal provided by the clock signal instead; because the clock signal is regenerated by averaging over many TDPPM pulses (flywheel effect), false pulses would have no influence on the state of the demultiplexer. However, due to the FS circuit, after a disturbance it is possible to recover proper synchronization within the duration of one single frame. Consequently, when using the TDPPM pulses themselves the influence of false pulses is limited.

The discussion of where to insert the demultiplexer, is very similar to the discussion of where to insert the multiplexer in the transmitter. Because of implementation considerations concerning the demultiplexer and the demodulator, we use one demodulator for each output and choose to demultiplex before demodulating.

The clock signal is regenerated by averaging the positions of the received TDPPM pulses. Practically, this function is performed by a phase lock loop (PLL). Since the averaging time is finite, i.e., the bandwidth of the PLL is nonzero, the positions of the particular pulses that are used for clock regeneration may not be modulated by signals having frequencies in the order of the PLL bandwidth or lower. Therefore particular pulses are reserved for the purpose of clock regeneration. As frame pulses, due to their slightly larger width than other TDPPM pulses, can be easily distinguished from other pulses, we prefer to use frame pulses for clock regeneration.

The fact that the frame pulses are used for clock regeneration, means that the frame pulses may not be modulated by low-frequency signals. In addition, since the frame pulses are slightly wider than other pulses and since the pulses should be kept within their time slots, the allowed peak-topeak pulse deviation of the frame pulses is a little smaller than that of other TDPPM pulses. For these two reasons, the characteristics of the channel assigned to transfer the frame pulses are somewhat different to the characteristics of the other channels.

\section{SYSTEM SPECIFICATIONS}

In this section we will determine the signal-to-noise ratio (SNR) and the maximum of the so called total signal bandwidth (denoted by $B_{\text {tot }}$ ) that can be achieved by the TDPPM system. $B_{\text {tot }}$ is defined as the product of the number of multiplexed signals and the bandwidth of the signals. These parameters depend on the characteristics of the optical components and on the characteristics of the receiver frontend, which is the most critical part of the electronic circuitry [11]. By making these dependencies explicit, the associated trade-offs become manageable. In addition, we will determine the threshold level below which the $\mathrm{SNR}_{0}$ collapses due to false pulses caused by a low value of the SNR of the received pulse signal $\left(\mathrm{SNR}_{r}\right)$. We will also discuss the influence of optical reflections on distortion and crosstalk and compare the $\mathrm{SNR}_{o}$ with the SNR achieved by a digital system.

\section{A. Low-Cost Optical Components}

As we wish to develop a low-cost system, we only consider using LED's or multimode lasers and PIN photodiodes. With regard to the performance of the TDPPM system the following characteristics of these components are important:

Laser diodes are superior to LED's with respect to the modulation bandwidth ( $1-4 \mathrm{GHz}$ compared to $20-200 \mathrm{MHz}$ ) and the power transmitted into the fiber $(0.5-2.5 \mathrm{~mW}$ com- 
pared to $50-500 \mu \mathrm{W})$. We therefore assume a laser diode to be used. The relative intensity noise (RIN, which may be regarded as the reciprocal of the carrier-to-noise ratio) of the laser light depends on the magnitude of the optical reflections received by the laser [7]. Since special measures preventing fiber reflections are not low cost, we have to accept reflections in the order of $2 \%$ resulting in an RIN of about $-125 \mathrm{~dB} / \mathrm{Hz}$. As a consequence, the laser noise determines the maximum SNR of the transmitted signals.

The minimal width $T_{p}$ of the TDPPM pulses that can be properly handled by lasers is in the order of $2 \mathrm{~ns}$. This value is rather large but we have to bear in mind that the spacing between the pulses must be large enough to settle the laser pulse tail close enough to its stationary value before the next pulse is supplied. Therefore $T_{p}$ incorporates the pulse tails.

PIN photodiodes achieve a bandwidth comparable to that of the laser, achieve high quantum efficiencies and contribute quantum determined noise.

As the transmission length in the aimed applications is relatively short, the losses of the fiber will be relatively low. If the transmission length is less than about $1 \mathrm{~km}$ the influence of wavelength dispersion is small compared to the influence of the bandwidth capabilities of the laser and the photodiode, so 680 or $850 \mathrm{~nm}$ components can be used. To keep mode dispersion acceptably low, we have to use (in order of increasing effectiveness) multimode fibers (up to about 100 $\mathrm{m}$ ), graded-index fibers (up to about $1 \mathrm{~km}$ ), or single mode fibers. However, the complexity of fiber coupling techniques, and thus cost, increases accordingly.

In the prototype system we used a multimode $1300 \mathrm{~nm}$ laser. Since the laser and PIN are mounted in optical connectors, different types of fiber can be attached. In addition, we carried out some successful experiments with low-cost (U.S. \$2) $680 \mathrm{~nm}$ lasers that are used in consumer CD players. As their mechanical positioning (in the package) is relatively inaccurate, they can only be combined with multimode fibers.

\section{B. Receiver Front-End}

The receiver front-end consists of a negative-feedback amplifier because it combines maximum sensitivity with the low input impedance required to avoid the loss of signal current into the parasitic capacitances of the PIN photodiode and the wiring between the PIN and the IC.

In this section, we consider the maximum bandwidth and the maximum sensitivity that can be achieved by integrated negative-feedback amplifiers. The maximal input current that can be handled by the amplifier will not be determined because we developed a method for enlarging the allowed magnitude of the input current of the front-end beyond that of the amplifier [9], [10].

Assuming that sufficient amplifier stages are used to obtain the required gain bandwidth product [8] (in our case we needed a two-stage amplifier), the bandwidth of negative-feedback amplifiers is restricted by the condition that the poles of the amplifier transfer function should be in specific (for instance Butterworth) positions. To locate the poles in Butterworth positions, the total phase shift within the loop should be $-90^{\circ}$ at the edge of the amplifier bandwidth. Other pole locations require the phase shift to be of the same order of magnitude. In a particular IC process, we examined the contributions of the (bipolar) transistors to the total phase shift and the possibilities of compensation by means of pole splitting and phantom zero's [11]. Because in a two stage amplifier a total phase shift of $-90^{\circ}$ can be established at a frequency of about $f_{t} / 10$ (in which $f_{t}$ denotes the transit frequency of the transistors), as a rule of thumb the maximum bandwidth of two-stage negative-feedback amplifiers (having for instance Butterworth pole locations) is in the order of $f_{t} / 10$. The bandwidth of amplifiers consisting of more than two stages is restricted to still lower frequencies.

In [9] we determined the total equivalent input noise current $I_{n}$ produced by a current-input amplifier in combination with a capacitive source impedance $C_{s}$, which consists of the capacitances of the PIN photodiode and the wiring between the PIN and amplifier. In accordance with [8], it is assumed that the amplifier has been designed such that only the first stage contributes to the amplifier noise. To find its fundamental minimum, the biasing and geometry of this transistor has been optimized. If $C_{j}$ is the total base-emitter and base-collector junction capacitance, $R_{\mathrm{b}}$ is the base bulk resistance and $\beta$ is the low-frequency current gain of the transistor, we find:

$$
I_{n}^{2} \approx \frac{8 \pi}{3} k T B_{r}^{2}\left[\left(C_{j}+C_{s}\right) \sqrt{\frac{3}{\beta}}+2 \pi B_{r} R_{b} C_{s}^{2}\right]
$$

in which $B_{r}$ denotes the receiver bandwidth.

As an example, we calculated the equivalent input noise current of an amplifier realized in the process used for the prototype system. Taking $B_{r}=250 \mathrm{MHz}, C_{s}=2 \mathrm{pF}$, $\beta=100, R_{b}=40 \Omega$ and $C_{j}=1.4 \mathrm{pF}$, we found $I_{n}=43$ nA. In a $10 \mathrm{GHz}$ process with $B_{r}=1 \mathrm{GHz}$ we found that $I_{n}=170 \mathrm{nA}$.

Because noise at frequencies beyond $B_{r}$ is not suppressed completely, (1) is only an approximation. The values of the noise currents of practical amplifiers are $1-3 \mathrm{~dB}$ higher.

\section{Signal-to-Noise Ratio and Total Signal Bandwidth}

Presently, our objectives are to calculate the output SNR $\left(\mathrm{SNR}_{o}\right.$ ) that can be achieved by an $N$-channel TDPPM system and to examine how the $\mathrm{SNR}_{o}$ depends on the ratio between the bandwidth of the transmitted signals and the total transmission bandwidth available. Before calculating the $\mathrm{SNR}_{o}$, we have to deal with the shape of the transmitted TDPPM pulses and the method used to reshape the received pulses.

1) Pulse Shaping and Pulse Reshaping: To achieve a high SNR, we have to minimize the jitter of the reshaped pulses and to maximize the maximal pulse deviation. In [11] we determined the optimum shape of the TDPPM pulses and the optimum pulse reshaping filter. At this stage, however, we delimit our discussion to a type of pulse that is practically feasible in a low-cost system. In addition, we use the low-pass filter function of the receiver amplifier.

The simplest laser driver just switches the laser on and off. As the rise and fall times of practical pulses of course are 


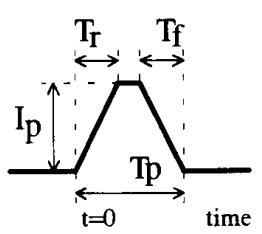

(a)

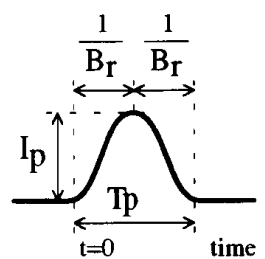

(b)
Fig. 4. (a) A trapezoid as an approximation of the waveform of optical pulses transmitted by an on-off laser driver. (b) A pulse having raised cosine edges as an approximation of the waveform of low-pass filtered TDPPM pulses.

finite, we assume that the shape of the TDPPM pulses can be approximated by a trapezoid, as depicted in Fig. 4(a). The rise and fall times will be denoted by $T_{r}$ and $T_{f}$ and the total pulse width by $T_{p} . I_{p}$ is the pulse amplitude.

If the bandwidth of the receiver amplifier $B_{r}$ is large compared to $1 / T_{r, f}$, the shape of the trapezoidal pulses is unaltered. In practical systems, this situation must be avoided because taking $B_{r}$ larger than necessary for handling the pulses only results in additional noise at the output of the filter, causing additional pulse jitter. If, on the other hand, $B_{r}$ is in the order of, or small compared to $1 / T_{r, f}$, the shape of the filtered pulses will be determined by the bandwidth and the precise pole locations of the receiver filter. If the order of the filter is 2 or higher, the filtered pulses will contain little energy at frequencies beyond $B_{r}$. In addition, because the maximal pulse deviation equals the slot length $T_{s l}$ minus the total pulse width $T_{p}$, we assume that $T_{p}$ is minimized to achieve a maximal pulse deviation. Then, the shape of the filtered pulses can be approximated reasonably well by pulses having the shape of a raised cosine, see Fig. 4(b):

$$
\begin{gathered}
P(t)=\frac{I_{p}}{2}\left[1+\cos \pi B_{r}\left(t-\frac{1}{B_{r}}\right)\right] \\
\text { if } \quad 0 \leq t \leq \frac{2}{B_{r}} .
\end{gathered}
$$

2) Pulse Jitter: The jitter in the positions of the reshaped pulses $\sigma_{t_{p}}$ is calculated by determining the ratio between the noise power and the squared slope magnitudes of the pulses at the filter output [to be determined from (2)]:

$$
\sigma_{t_{p}}^{2}=\frac{I_{n}^{2}}{\frac{I_{p}^{2} \pi^{2} B_{r}^{2}}{4}} .
$$

Thereby we assumed that the root mean square magnitude of the noise is small compared to the amplitude of the pulses and that the noise is additive to the received PPM pulse signal. Although the later assumption does not apply for the noise produced by the laser and the photodiode, it leads to a reasonable approximation of the resulting SNR.

To write $\sigma_{t_{p}}$ as a function of the $\mathrm{SNR}_{r}$ of the received (TD)PPM pulses, we define:

$$
\mathrm{SNR}_{r}={ }_{\text {def }} \frac{\left(\frac{I_{p}}{2}\right)^{2}}{I_{n}^{2}} .
$$

The $\mathrm{SNR}_{r}$ is defined at half the pulse height because it is the decision level of the comparator. The numerator of (4) is a measure of the power of the TDPPM pulses, further denoted by $P_{r}$. The denominator denotes the mean square value of the noise.

Depending on $P_{r}$ we distinguish two important contributions to the $\left.\mathrm{SNR}_{r}: 1\right)$ if $P_{r}$ is small the $\mathrm{SNR}_{r}$ depends on the noise current contributed by the receiver front-end given by (1) so the $\mathrm{SNR}_{r}$ is proportional to $P_{r}$, and 2) if $P_{r}$ is relatively large the $\mathrm{SNR}_{r}$ is limited by the RIN of the laser diode. Since the RIN equals the ratio between the noise power density and the $\mathrm{dc}$ power the maximum of the $\mathrm{SNR}_{r}$ is given by

$$
\mathrm{SNR}_{r}=\frac{1}{\mathrm{RIN} B_{r}} .
$$

These approximations are realistic if the order of the filter is two or higher. Because of the relatively poor RIN of low-cost lasers, the shot noise produced by the PIN photodiode can be neglected in all cases.

Assuming an RIN level of $-125 \mathrm{~dB} / \mathrm{Hz}$ while $I_{n}=43 \mathrm{nA}$ for $B_{r}=250 \mathrm{MHz}$ and $I_{n}=170 \mathrm{nA}$ for $B_{r}=1 \mathrm{GHz}$ the take-over levels are at $I_{p}=9.4 \mu \mathrm{A}$ and $I_{p}=19 \mu \mathrm{A}$ respectively.

Using (4), (3) can be rewritten as:

$$
\sigma_{t_{p}}^{2}=\frac{1}{\operatorname{SNR}_{r} \pi^{2} B_{r}^{2}}
$$

3) Dependence of the Signal-to-Noise Ratio on the Pulse Jitter: The SNR depends on the peak-to-peak pulse deviation $2 T_{\Delta}$ and the jitter in the decision time $\sigma_{t_{p}}$ of the pulse reshaper. As the demodulator converts the positions of the (TD)PPM pulses directly into the momentary amplitude of the output signal, the SNR of the demodulator output signal $\left(\mathrm{SNR}_{d}\right)$ follows directly from $T_{\Delta}$ and $\sigma_{t_{p}}$ :

$$
\mathrm{SNR}_{d}=\frac{\frac{T_{\Delta}^{2}}{2}}{\sigma_{t_{p}}^{2}}
$$

in which we substituted the "effective signal power" $T_{\Delta}^{2} / 2$. Thereby the peak-to-peak deviation of the (TD)PPM pulses, which equals the length of the time slots $T_{s l}$ minus the pulse width $T_{p}$, can be written as:

$$
\begin{aligned}
2 T_{\Delta} & =\frac{1}{N f_{s}}-T_{p} \\
f_{s} & <\frac{1}{N T_{p}}
\end{aligned}
$$

where $N$ is the number of PPM signals multiplexed into one TDPPM signal (the number of slots in a frame, see Fig. 1) and $f_{s}$ is the sampling frequency of one PPM signal. The condition given by (9) excludes negative values of $T_{\Delta}$.

At frequencies higher than $f_{s} / 2$ the frequency spectrum of the demodulator output signal only contains repetitions, so the maximum bandwidth of the baseband signal amounts to $f_{s} / 2$. Because finite-order smoothing filters will be used, the actual bandwidth of the transmitted signals $B_{l}$ can not cover the 
potentially available baseband bandwidth. With regard to this so-called over sampling, we define the over sampling ratio as:

$$
r=\frac{f_{s}}{2 B_{l}} \text {. }
$$

Practical values of $r$ are in the range of 1.5-2.

The consequence of over sampling is that the smoothing filter will suppress the noise between $B_{l}$ and $f_{s} / 2$. Hence, the $\mathrm{SNR}_{o}$ will be larger than the $\mathrm{SNR}_{d}$ :

$$
\mathrm{SNR}_{u}=\mathrm{SNR}_{d} \frac{f_{s}}{2 B_{l}}=\mathrm{SNR}_{d} r .
$$

Substitution of (8) and (6) into (7) and multiplying by $r$ yields:

$$
\mathrm{SNR}_{o}=\operatorname{SNR}_{r}\left(\frac{1}{2 N r B_{l}}-T_{p}\right)^{2} \frac{\pi^{2} B_{r}^{2}}{8} r .
$$

Evaluation of (12), while bearing in mind the condition of (9), shows that $\mathrm{SNR}_{o}$ increases with decreasing $r$. Obviously, the appropriate value of $r$ is a compromise between optimal performance $(r=1)$ and the cost of the smoothing filter, requiring larger values of $r$.

Equation (12) gives the SNR as the ratio between the mean signal power and the mean noise power. In the particular case of video signals, the video SNR is defined as the ratio between the square of the black-to-white amplitude and the mean noise power, yielding a $6 \mathrm{~dB}$ higher value. Since the noise spectrum at the demodulator output is flat, the weighted video SNR (for PAL coded video signals) is approximately another $6 \mathrm{~dB}$ higher.

4) Dependence of the Signal-to-Noise Ratio on the Total Signal Bandwidth: Some interesting results are obtained if the relation between $\mathrm{SNR}_{o}$ and $\mathrm{SNR}_{r}$, given by (12), is considered as a function of the ratio between the so-called total signal bandwidth $B_{\text {tot }}$ and the receiver bandwidth $B_{r}$. The total signal bandwidth $B_{\text {tot }}$ is defined as the product of the number of multiplexed signals $N$ and the available bandwidth per channel $B_{l}$ :

$$
B_{\text {tot }}=\text { def } N B_{l} \text {. }
$$

Hence, (12) can be rewritten as:

$$
\mathrm{SNR}_{o}=\mathrm{SNR}_{r}\left(\frac{1}{4 r B_{\text {tot }}}-\frac{T_{p}}{2}\right)^{2} \frac{\pi^{2} B_{r}^{2}}{2} r .
$$

The $\mathrm{SNR}_{o}$ yields zero if the maximum pulse deviation yields zero, which is the case if $B_{\text {tot }}$ becomes so large that the related repetition frequency of the TDPPM pulses (i.e., $2 N r B_{l}$ ) equals the reciprocal of the width of the TDPPM pulses $1 / T_{p}$. Therefore, we define the maximum of the total signal bandwidth $B_{\text {tot }}$ as:

$$
B_{\text {tot }, \max }=\frac{1}{2 r T_{p}}
$$

In Section IV-A we concluded that the laser diode limits the pulse width to a minimum of 2 ns. Hence, if we assume $r=1.75$, we conclude that the laser diode limits $B_{\text {tot, } \max }$ to about $140 \mathrm{MHz}$. A second factor limiting the pulse width is the electronic circuitry where the bandwidth of the receiver

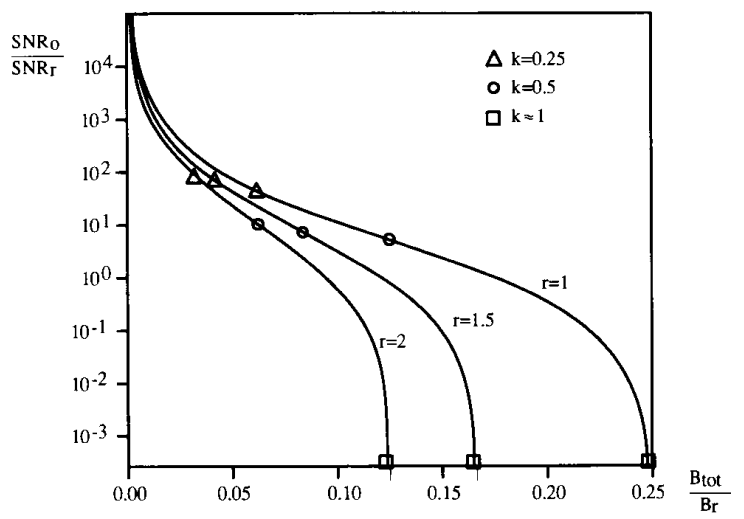

Fig. 5. Ratio between SNRo and SNR, versus ratio between $B_{\text {t.ot }}$ and $B_{r}$ for different values of $r$. The factor $k$ denotes the ratio between $B_{l, t}$ and $B_{101, \max }$

amplifier is the most critical. In the practical case where the $f_{t}$ of the IC process is below $10 \mathrm{GHz} T_{p}$ is determined by the bandwidth $B_{r}$ of the receiver amplifier. Using the raised cosine approximation given in (2), we find:

$$
B_{\mathrm{tot}, \text { max }}=\frac{B_{r}}{4 r} \text {. }
$$

Finally, by combining (14) and (16) and by defining $k$ as $B_{\text {tot }} / B_{\text {tot,max }}$ we may write:

$$
\mathrm{SNR}_{o}=\mathrm{SNR}_{r} \frac{(k-1)^{2}}{k^{2}} \frac{\pi^{2}}{2} r .
$$

Fig. 5 depicts three curves calculated from (17) for different values of $r$. The curves demonstrate the tradeoff between bandwidth and $\mathrm{SNR}_{o}$. To achieve a sufficiently high $\mathrm{SNR}_{o}$, practical values of $k$ are in the order of 0.8 or lower. It shows that for the highest $\mathrm{SNR}_{o}$ and the largest $B_{\text {tot,max }} r$ should preferably be close to unity.

5) Characteristics of the Experimental System: In an experimental system implemented in a $2.5 \mathrm{GHz}$ process we realized $B_{r}=250 \mathrm{MHz}$ (about $f_{t} / 10$, see Section IV-B), $r=1.75$ and $k=0.55$, which makes possible the transmission of 4 standard PAL coded video signals ( $B_{\text {tot }}=20 \mathrm{MHz}$ ). In a $10 \mathrm{GHz}$ process $N=16$ is feasible.

Fig. 6 shows the $\mathrm{SNR}_{o}$ given by (17) whereby the $\mathrm{SNR}_{r}$ depends on $P_{r}$. It has been assumed that $r=1.75$ and $k=0.55$. For the sake of completeness, the $X$-axis has alsobeen calibrated in terms of the optical input-power level (pulse top). The sensitivity of the PIN diode has been assumed to be $0.8 \mathrm{~A} / \mathrm{W}$.

\section{False-Pulse Threshold Level}

Up to now we assumed that only the positions of the pulses are changed. However, if the momentary amplitude of the noise may (temporarily) exceed half the pulse height, false pulses will result at the reshaper output or intended pulses will be missed.

Assuming a Gaussian distribution of possible momentary noise amplitudes the chance that the noise voltage (having an 


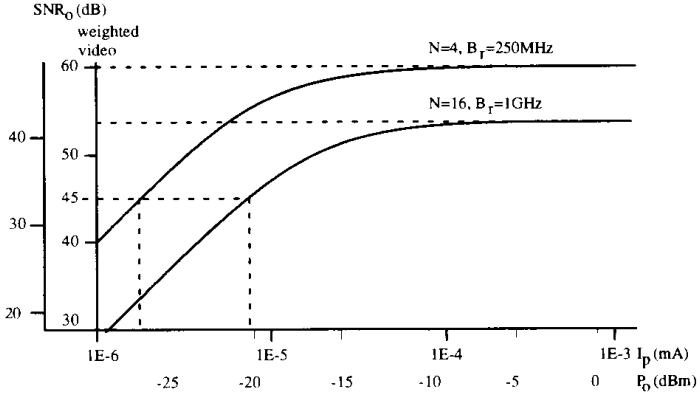

Fig. 6. SNR (weighted for PAL coded video) as a function of the received optical power (pulse top) for $Y=4\left(B_{r}=250 \mathrm{MHz}\right.$, upper trace) and $\mathrm{V}=16\left(B_{r}=1 \mathrm{GHz}\right.$, lower trace $)$. In both cases $B_{l}=5 \mathrm{MHz}$.

average power $I_{n}^{2}$ ) at any time exceeds half the pulse height is given by (if $\mathrm{SNR}_{r} \gg 1$ ):

$$
P\left(i_{n}>\frac{I_{p}}{2}\right) \approx \frac{1}{\sqrt{2 \pi \mathrm{SNR}_{r}}} \exp -\frac{\mathrm{SNR}_{r}}{2}
$$

in which we used the approximation of the error function given in $[13$, p. 666].

If the bandwidth of the noise equals $B_{r}$, according to Nyquist the received signal can be described by $2 B_{r}$ samples per second. Hence, the average time between two false pulses is calculated to be

$$
T_{f p}=\frac{\sqrt{2 \pi \mathrm{SNR}_{r}} \exp \frac{\mathrm{SNR}_{r}}{2}}{2 B_{r}} .
$$

The value of $T_{f p}$ that is just acceptable depends on the application. For example, in the case of analog video signals the disturbances will be quite acceptable if $T_{f p}$ is in the order of a second. If $B_{r}$ is $250 \mathrm{MHz}$ (as in the prototype system), this value requires an $\mathrm{SNR}_{r}$ of about $15 \mathrm{~dB}$.

Due to the exponential term in (19), the required $\mathrm{SNR}_{r}$ is not very sensitive to the desired value of $T_{f p}$. Hence we may speak of a threshold value of the $\mathrm{SNR}_{r}$ below which the $\mathrm{SNR}_{o}$ of the received signals rapidly decreases. In addition, also due to the exponential relation, the required $\mathrm{SNR}_{r}$ does not depend very much on $B_{r}$. So, as a rule of thumb, we may further assume the threshold to be at an $\mathrm{SNR}_{r}$ of about $15 \mathrm{~dB}$.

\section{E. Influence of Optical Reflections}

In the ideal case, all optical power arriving at the receiver is absorbed by the photodiode. However, in practical systems a small fraction $r_{2}$ is reflected back to the transmitter, see Fig. 7. When the reflected pulses arrive at the transmitter, a fraction $r_{1}$ of their power is reflected for the second time. Consequently the photodiode receives delayed and doubly reflected TDPPM pulses, the so-called triple transit echo, in addition to the intended pulses. As the echoes virtually modulate the positions of the intended pulses, small distortions and crosstalk between signals will result.

In addition, a fraction of the pulses that are reflected once will enter the cavity of the laser diode and interfere with the transmitted pulses. Due to the nonlinearities of the lasing mechanism, the modeling of this effect is difficult. To estimate

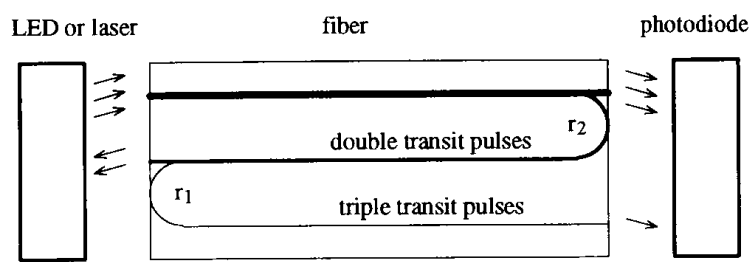

Fig. 7. Reflections at the interfaces of laser/fiber and fiber/photo diode.

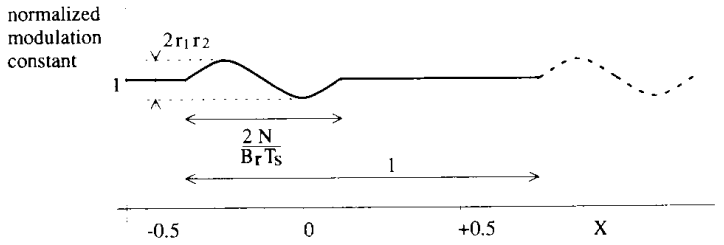

Fig. 8. Normalized gain as a result of the presence of triple transit pulses.

the influence of the double transit pulses, measurements are necessary.

In [11] it is shown that the presence of triple transit pulses results in modulation of the gain of the transmitted signals. Thereby, we considered the worst case situation, which is when the optical losses are small. Assuming the shape of the TDPPM pulses at the output of the receiver amplifier to be that of a raised cosine, we found that the normalized gain depends on the momentary amplitude of the input signal $X$ as shown in Fig. 8. The curve has the shape of the time derivative of the raised cosine pulses. Depending on the transmission delay time the curve can shift along the $X$-axis. So, the level of $X$ for which the distortion occurs may vary.

By defining the differential gain (DG) as

$$
\mathrm{DG}={ }_{\text {def }} \frac{\text { gain }_{\text {max }}-\text { gain }_{\text {min }}}{\text { gain }_{\text {average }}}
$$

it clearly shows from Fig. 8 that the differential gain caused by the triple transit pulses equals

$$
\mathrm{DG}=2 r_{1} r_{2}
$$

Practical values of the reflection coefficients $r_{1}$ and $r_{2}$ are in the order of $2 \%(-17 \mathrm{~dB}$, in optical power), so the DG is practically negligible (in the order of $0.1 \%$ ).

The second consequence of pulse reflection is crosstalk. By defining the crosstalk $C$ as the ratio between the peak-to-peak deviation of $t_{d}$ caused by the triple transit pulses and the peak-to-peak deviation due to modulation, it was shown that:

$$
C=\frac{r_{1} r_{2}}{\pi} \frac{k}{1-k}
$$

The crosstalk is calculated to be in the range of -78 to -66 $\mathrm{dB}$.

\section{F. Comparison with Digital Coding}

Now, we compare the performance of the TDPPM system with that of an alternative system based on digital coding. We assume an $N$ channel digital system, exhibiting the same transmission bandwidth $B_{r}$, baseband bandwidth $B_{l}$ and over 
sampling factor $r$ as the TDPPM system. We assume no bits are lost due to noise or triple transit pulses.

The SNR, which does not depend on the received power level, is calculated to be:

$$
\mathrm{SNR}_{\mathrm{dig}}=\frac{3}{2} 2^{2 n} r
$$

Thereby, the $\mathrm{SNR}_{\text {dig }}$ is assumed to be determined by the quantization noise [13], pp. 434-435. The parameter $n$ denotes the number of bits that is available for each sample. $n$ equals the maximal transmission bit rate $2 B_{r}$ divided by the number of samples $2 N B_{l} r$ (of all channels together) per unit of time:

$$
n=\frac{2 B_{r}}{2 N B_{l} r}=\frac{4}{k} \text {. }
$$

Taking $r=1.75$ and $k=0.55$ yields $\mathrm{SNR}_{\mathrm{dig}}=48 \mathrm{~dB}$. The weighted video SNR amounts to $60 \mathrm{~dB}$.

Obviously, if $P_{r}$ is in the range where the noise produced by the laser diode dominates, the digital system achieves about the same SNR as the TDPPM system. With respect to the tradeoff between $B_{\text {tot }}$ and SNR, we found that the TDPPM system is superior when the emphasis is on a large $N$. On the other hand, when the emphasis is on a high SNR, a decreasing $B_{\text {tot }}$ yields more bits per sample. Since each bit results in an SNR which is $6 \mathrm{~dB}$ higher, in these cases digital systems outperform TDPPM. Provided that no bits are lost, in the digital system, triple transit pulses do not cause distortion and crosstalk.

In low-cost applications high SNR's are not required. However, having a large number of channels helps to reduce costs. Obviously, for low-cost applications the TDPPM system is the best choice.

\section{EXPERIMENTAL RESULTS}

A four-channel experimental set-up has been realized in a $2.5 \mathrm{GHz}$ semicustom bipolar process. We used the Fujitsu FLD130C2PM multimode laser diode mounted in an FC connector with a maximum power of $-3 \mathrm{dBm}$, a Fujitsu photodiode, graded index/single mode fiber and a variable optical attenuator.

Measurements made on the VM700 revealed a differential gain (DG) of $0.5 \%$ and a differential phase below $0.1^{\circ}$ (Fig. 9). The measured value of the DG cannot be explained by our calculations. However, the shape of the normalized gain curve is similar to the curve depicted in Fig. 8 while replacing the optical fiber by another piece of fiber resulted in a different DG. In addition, separate measurements of the distortion of the modulator and demodulator revealed considerably lower values. So, we figured that the distortion in the prototype system was caused by the (once reflected) double transit pulses, which interfere with the pulses that are transmitted by the laser diode. It was concluded earlier that the modeling of this effect is difficult. Although crosstalk was not observed, since the mechanisms causing crosstalk and distortion are the same, the crosstalk will be higher than the value given by (22).

To avoid the interference of double transit pulses, we have to use better fiber connection techniques or insert an optical isolator between the laser diode and the fiber. Unfortunately,

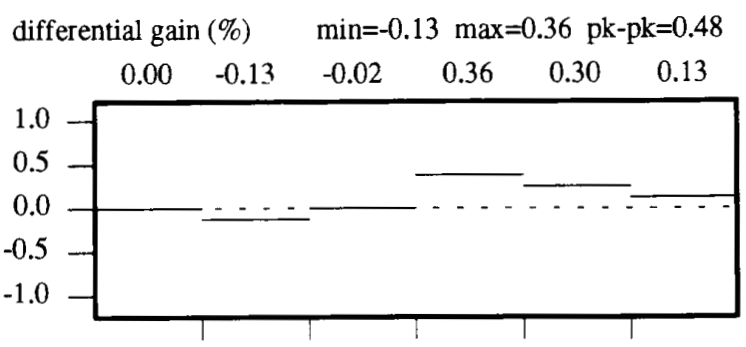

differential phase $(\mathrm{deg}) \quad \min =-0.05 \max =0.02 \mathrm{pk}-\mathrm{pk}=0.07$

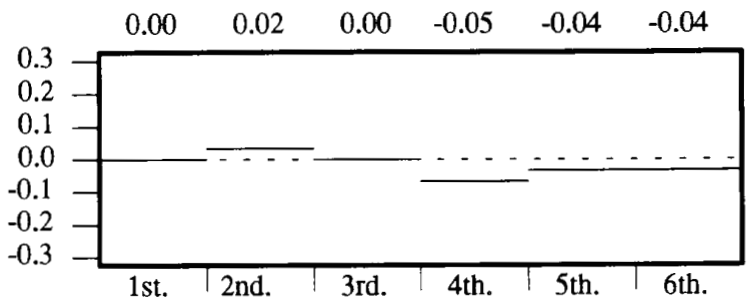

Fig. 9. Differential gain and phase measured by the VM700.

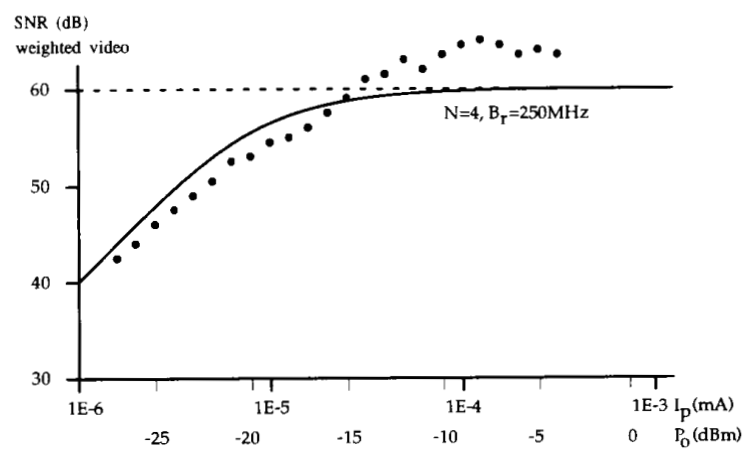

Fig. 10. Measured and calculated SNR (weighted) as a function of the received optical power (pulse amplitude) in the four-channel version.

these solutions are not low cost. Once more, there is a trade off between quality and cost.

Fig. 10 depicts the calculated and the measured weighted SNR as a function of the received power level (pulse amplitude). For low power levels, the calculated and the measured values are in close agreement. For higher power levels, where laser noise dominates, the measured SNR was better than the calculated SNR, probably since the RIN was better than we assumed in our calculations. The relatively large spread in the measured SNR's at high power levels is caused by the influence of reflections on the RIN. The reflection properties depend on the actual settings of the variable attenuator.

At short distances, the influence of reflection and coupling can be reduced by using multimode fiber. In this case a slightly higher SNR and a lower distortion were observed. This is because reflections back to the laser cavity can be reduced by using multimode rather than singlemode fiber.

\section{CONCLUSIONS}

The best strategy for the low-cost transmission of video signals over distances up to a few kilometers consists of two 
components: 1) Using a low-cost laser diode and integrating the electronic circuitry in a low-cost standard IC process to minimize the costs of the transmitter and receiver and 2) applying multiplexing techniques to reduce the relative costs of the fiber, common circuitry and installation.

TDPPM appeared to be the most appropriate type of coding since its implementation requires less chip area and consumes less supply power than the digital alternatives. Moreover, for typical low-cost applications where the emphasis is on the number of channels and not on the SNR, TDPPM coding performs better than digital coding.

We calculated that in a $10 \mathrm{GHz}$ process 16 channels can be multiplexed with a weighted SNR of $56 \mathrm{~dB}$ (for PAL coded video). For a weighted SNR of $45 \mathrm{~dB}$ and a launched power of $0 \mathrm{dBm}$ the optical budget exceeds $20 \mathrm{~dB}$. The required chip area is about $5 \mathrm{~mm}^{2}$ per transmitter/receiver. When external video output buffers are used, the power consumption amounts to $1 \mathrm{~W}$.

The feasibility of the system was successfully tested for a four-channel version in a $2.5 \mathrm{GHz}$ bipolar semicustom process. Measurements revealed a differential gain below $1 \%$ and a differential phase below $0.5^{\circ}$.

It can be concluded that the proposed TDPPM transmission system fully satisfies the requirements for the intended lowcost, multichannel applications.

\section{REFERENCES}

[1] L. P. de Jong, E. H. Nordholt, and C. M. C. J. Hooghiemstra, "High performance integrated receiver circuits for optical fiber transmission of wideband FM video signals," IEEE Trans. Consumer Electron., vol. CE-33, pp. 473-480, Aug. 1987.

[2] S. F. Heker, G. J. Herskowitz, H. Grebel, and H. Wichansky, "Video transmission in optical fiber communication systems using pulse frequency modulation," IEEE Trans. Commun., vol. 36, pp. 191-194, Feb. 1988.

[3] S. Y. Suh, "Pulse width modulation for analog fiber-optic communications," J. Lightwave Technol., vol. LT-5, pp. 102-112. Jan. 1987.

[4] T. E. Darcie, "Subcarrier multiplexing for lightwave networks and video distribution systems," IEEE J. Select. Areas Commun., vol. 8, pp. 1240-1248, Sept. 1990.

[5] J. A. Chiddix, H. Laor, D. M. Pangrac, L. D. Williamson, and R. W Wolfe, "AM video on fiber in CATV systems: Need and implementation," IEEE J. Select. Areas Commun., vol. 8, pp. 1229-1239, Sept. 1990.

[6] M. Maeda and M. Yamamoto, "FM-FDM optical CATV transmission experiment and system design for MUSE HDTV signals," IEEE $J$ Select. Areas Commun., vol. 8, pp. 1257-1267, Sept. 1990.

[7] K. Petermann, Laser Diode Modulation and Noise. Dordrecht, The Netherlands: Kluwer-Academic, 1991

[8] E. H. Nordholt, Design of High-Performance-Feedback Amplifiers. Amsterdam, The Netherlands: Elsevier-Scientific, 1983

[9] L. A. D. van den Broeke and A. J. Nieuwkerk, "Wide-band integrated optical receiver with improved dynamic range using a current switch at the input," IEEE J. Solid-State Circuits, vol. 28, pp. 862-864, July 1993.

[10] "High-dynamic range wide-band integrated receiver for optical transmission using a gain switch at the input," in Eighteenth European Solid State Circuits Conf., Sept. 21-23, 1992, pp. 287-290.

[11] L. A. D. van den Broeke, "A single-chip multi-channel optical transmission system," Ph.D dissertation, Delft Univ. Technol., 1994

[12] J. P. Moree, G. Groenewold, and L. A. D. van den Broeke, "A bipolar integrated continuous-time filter with optimized dynamic range," IEEE J. Solid-State Circuits, vol. 28, pp. 954-961, Sept. 1993
[13] A. B. Carlson, Communication Systems: An Introduction to Signals and Noise in Electrical Communication. New York: McGraw-Hill, 1968. p. 297.

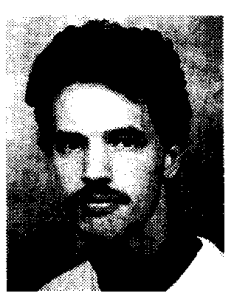

L. A. Dick van den Broeke was born in Middelharnis, The Netherlands, on January 19,1966. He received the M.Sc. degree in 1989 from the Delft University of Technology in electrical engineering He received the Ph.D. degree in February 1994 from the same faculty on the subject of integrated circuits for low-cost optical transmission systems.

Presently, he works as analog designer in the Consumer IC's Group of Philips Semiconductors in Nijmegen, the Netherlands.

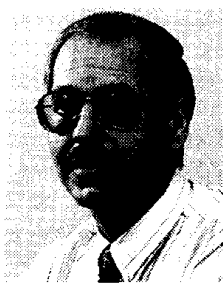

Leo P. de Jong was born in Bolsward, The Nether lands, on April 2, 1945. He received the M.Sc. degree in electrical engineering from the Delft University of Technology in 1969.

Since 1971, he has been a member of the scientific staff of the Electronics Research Laboratory, where he is presently an Associate Professor. His research interests include analog circuit design with emphasis on applications in the field of sensor interfacing and integrated radio and fiber-optic communication systems.

Jan Davidse received the M.Sc. degree in electrical engineering from the Delft University of Technology in 1953 and the Ph.D. degree from the Eindhoven University of Technology.

He was a research scientist with Philips Research Laboratories and received a full professorship in electronics at the Delft University of Technology in 1964. Since 1964, he has been the Head of the Laboratory for Electronics at this university. His research interest is in analog electronics and in electronic pictorial techniques. He is the author of numerous papers and holds several patents in his field of interest. He is the author of 10 books, among which is Analog Electronic Circuit Design (Englewood Cliffs, NJ: Prentice-Hall, 1991).

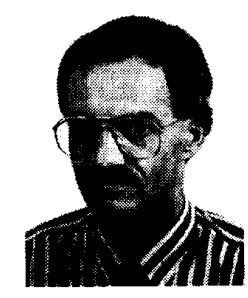

Arthur H. M. van Roermund was born in Delft, The Netherlands in 1951. He received the M.Sc. degree in electrical engineering in 1975 from the Delft University of Technology and the Ph.D. degree in applied sciences from the K.U. Leuven, Belgium, in 1987.

From 1975 to 1992 he was with the Philips Research Laboratories in Eindhoven. First he joined the Consumer Electronics Group, where he has been involved with the design and integration of analog circuits and systems, especially switched-capacitor circuits. In 1987 he joined the Visual Communications Group where he has been engaged in video architectures and digital video signal processing. From 1987 to 1990 he was project leader of the Video Signal Processor project and from 1990 to 1992 of a Multiwindow Television project. Since 1992 he has been a full professor at the Electrical Engineering Department of the Delft University of Technology. He heads the Electronics Laboratory, which is part of DIMES: The Delft Institute of Micro Electronics and Submicron technology. 